\title{
La Nueva Ola Feminista y las luchas de las mujeres trabajadoras ¿Por qué luchamos?
}

\author{
Paula Varela ${ }^{1}$
}

\begin{abstract}
Resumo
Durante los últimos 5 años hemos sido testigos de una Nueva Ola Feminista a nivel mundial marcada por dos elementos que le otorgan características particulares: a) La crisis del capitalismo neoliberal que comienza en 2008 y su expresión en planes de ajuste, aumentos de precarización laboral, incremento de la pobreza y del desempleo. La nueva ola feminista forma parte de los movimientos de protesta surgidos al calor de la crisis. b) La importancia que asume en él la figura del trabajo que realizan las mujeres en la sociedad capitalista contemporánea, esto es, el rol de las mujeres en el trabajo de reproducción social. La Huelga internacional de Mujeres, como principal instancia de articulación del movimiento a nivel internacional, marca la centralidad de este elemento de clase en el movimiento de mujeres y marca, al mismo tiempo, la centralidad de las mujeres en la clase que mueve el mundo. En este artículo analizamos la relación entre el ascenso del movimiento de mujeres y las demandas de las mujeres trabajadoras a partir de cuatro procesos huelguísticos que presentan la particularidad de desarrollarse en el lugar de trabajo y ser liderados por mujeres.
\end{abstract}

\section{Palabras clave}

Feminsimo; clase trabajadora; reproducción social; neoliberalismo; crisis.

The New Feminist Wave and Working Women`s Struggles: What are we fighting for?

\begin{abstract}
During the last five years we have witnessed a New Feminist Wave worldwide characterized by two elements: a) The crisis of neoliberal capitalism that began in 2008 and its expression in austerity plans, increases in the precariousness of the labor market, and increases in poverty and unemployment. The New Feminist Wave is part of the protest movements that have emerged in the heat of the crisis. b) The importance that the work that women do in contemporary capitalist society has taken in it, that is, the role of women in social reproduction work. The International Women's Strike, as the main articulation instance of the movement at international level, marks the centrality of this class element in the women's movement and at the same time it marks the centrality of women in the class that moves the world. In this article, we analyze the relationship between the rise of the women's movement and the women workers claims through four strike events that have the particularity of being developed in the workplace and being led by women.
\end{abstract}




\section{Keywords}

Feminism; working class; social reproduction; neoliberalism; crisis.

\section{Resumo}

Assistimos nos últimos 5 anos a uma Nova Onda Feminista em todo o mundo marcada por dois elementos que lhe conferem características particulares: a) A crise do capitalismo neoliberal iniciada em 2008 e sua expressão em planos de ajuste, aumento da precarização do emprego, aumento da pobreza e do desemprego. A nova onda feminista faz parte dos movimentos de protesto que surgiram no calor da crise. b) A importância que nela assume a figura do trabalho realizado pelas mulheres na sociedade capitalista contemporânea, ou seja, o papel da mulher no trabalho de reprodução social. A Greve Internacional das Mulheres, como principal instância de articulação do movimento em nível internacional, marca a centralidade desse elemento de classe no movimento feminista e marca, ao mesmo tempo, a centralidade das mulheres na classe que move o mundo. Neste artigo analisamos a relação entre a ascensão do movimento de mulheres e as demandas das mulheres trabalhadoras a partir de quatro processos de greve que apresentam a particularidade de serem realizadas no local de trabalho e lideradas por mulheres.

\section{Palavras-chave}

Feminismo; classe trabalhadora; reprodução social; neoliberalismo; crise.

Artigo recebido em junho 2020

Artigo aprovado em agosto de 2020

\section{La Nueva Ola Feminista y las luchas de las mujeres trabajadoras ¿Por qué luchamos?}

Durante los últimos 5 años hemos sido testigos de una Nueva Ola Feminista a nivel mundial. Argentina irrumpió con el reclamo de \#Niunamenos en 2015 como grito que exigía el fin de los femicidios y tres años después, en un movimiento de mujeres que se volvió cada vez más masivo y con fuerte presencia juvenil, pintó las calles de verde en reclamo del derecho al Aborto Legal Seguro y Gratuito. Del otro lado del Atlántico, las mujeres también ganaban las calles por el derecho al aborto, en Irlanda para conquistarlo luego de las luchas que derivaron en el histórico referéndum de mayo de 2018, en Polonia para no perderlo en un contexto en el que estaba siendo atacado. Del aborto se pasó a discutir la paridad salarial, la violencia machista y el trabajo 
doméstico. Estados Unidos viralizó su \#MeToo en 2017, al inicio como un movimiento reducido a las "celebrities" pero que luego derramó en otros sectores sociales, politizó a las mujeres y las transformó en uno de los principales actores movilizados contra la presidencia de Donald Trump. En similar sintonía, el movimiento de mujeres comenzó a crecer en Brasil y volverse visible al calor de la consigna "Ele nao" en referencia a la candidatura de quien sería finalmente presidente de la nación en 2019, Jair Bolsonaro. Italia vivió las movilizaciones más masivas de los últimos tiempos en la celebración de la Huelga Internacional de Mujeres del 8 de Marzo y la revitalización del histórico movimiento feminista de ese país a partir de la conformación de la plataforma de mujeres “Non di una Meno". En el Estado Español, el 8M se transformó también en la fecha clave de un movimiento masivo que desbordó las expectativas, y que recolocó el debate feminista en la agenda política nacional, haciendo que por ejemplo en Andalucía, el movimiento de mujeres fuera un actor político central contra el partido de extrema derecha Vox. Chile vivenció un ascenso del movimiento de mujeres que fue clave en las luchas estudiantiles en las que ganó peso la consigna por la educación sexual, y en las masivas manifestaciones desatadas por el aumento de la tarifa del transporte en 2019 pero que, como sus propios protagonistas se ocuparon de decir, no eran protestas "por 30 pesos" sino "por treinta años de neoliberalismo".

En síntesis, de Argentina a Polonia, de Estados Unidos a Chile, del Estado Español a Brasil (por mencionar sólo algunos países), esta nueva ola feminista se despliega en diferentes contextos sociales y políticos nacionales en los cuales coloca en el centro de la agenda pública temas como los femicidios y la violencia de género, los derechos reproductivos, las libertades sexuales, la brecha salarial y el trabajo doméstico. Pero la heterogeneidad de demandas y contextos no puede hacer perder de vista un elemento que atraviesa las distintas experiencias locales y que dota de características particulares a esta nueva ola: la crisis del capitalismo neoliberal que comienza en 2008 y su expresión 
en planes de ajuste, aumentos de precarización laboral, incremento de la pobreza y del desempleo. La nueva ola feminista forma parte de los movimientos de protesta surgidos al calor de la crisis. Como señala Cinzia Arruzza, "la explosion del movimiento feminista fue precedida por otras movilizaciones, como el ciclo de luchas de 2011-2013 que cobró visibilidad internacional (en particular Occupy, los Indignados y Plaza Taksim), con el cual presenta elementos de continuidad" (ARRUZZA, 2018)2. Este contexto hace que, en aquellos países en los que ha emergido masivamente, el movimiento de mujeres pierda su carácter sectorial y se transforme, fácilmente, en un fenómeno político que integra la pléyade de movimientos que luchan contra el ajuste, contra la derecha y contra las políticas autoritarias.

Pero hay otro aspecto que caracteriza esta Nueva Ola Feminista: la importancia que asume en él la figura del trabajo que realizan las mujeres en la sociedad capitalista contemporánea, esto es, el rol de las mujeres en el trabajo de reproducción social. "Si paramos nosotras, se para el mundo" puede leerse entre las consignas del 8 de Marzo. La Huelga internacional de Mujeres, como principal instancia de articulación del movimiento a nivel internacional, marca la centralidad de este elemento de clase en el movimiento de mujeres y marca, al mismo tiempo, la centralidad de las mujeres en la clase que mueve el mundo. La Huelga Internacional de Mujeres es la herramienta de las mujeres en tanto "trabajadoras esenciales" y miembros fundamentales de la clase trabajadora.

En este artículo analizamos la relación entre el ascenso del movimiento de mujeres y las luchas que llevan adelante las mujeres trabajadoras en el contexto de crisis del capitalismo neoliberal, teniendo en cuenta tres consideraciones. La primera, la perspectiva de la reproducción social (o la teoría de la reproducción social) como un abordaje que nos permite comprender con más profundidad el rol de liderazgo de las mujeres en las luchas de la clase trabajadora. La segunda, que cuando hablamos de reproducción social no estamos refiriéndonos únicamente a lo que sucede en el ámbito del hogar 
o el ámbito comunitario (barrios, comunidades) sino también a lo que sucede en miles de lugares de trabajo en los que, principalmente mujeres, llevan adelante el trabajo reproductivo (hospitales, escuelas, servicios de limpieza, etc.) $)^{3}$. Tener en cuenta estas dos formas del trabajo de reproducción social (el no pago y el asalariado), es fundamental para comprender la centralidad de las mujeres en la morfología de la clase trabajadora contemporánea ${ }^{4}$. Pero además, es fundamental porque las instituciones de reproducción social han sido uno de los principales focos de ataque del capitalismo neoliberal a través de privatizaciones y recortes presupuestarios, como sucedió desde la década del 90 en países del sur global (como Argentina), y más recientemente, en países europeos como Grecia, Italia, Portugal o España. La tercera consideración que es importante tener en cuenta es que el abordaje de la reproducción social nos permite poner el foco en las luchas de mujeres trabajadoras en tanto luchas que exceden las demandas económicas o estrictamente laborales, e introducen reclamos relacionados con necesidades fundamentales para la vida de la clase obrera que están siendo violentamente atacadas por el capital y las clases dominantes en los diferentes países.

Desde este punto de vista, presentaremos cuatro tipos de luchas desarrolladas en el lugar de trabajo y lideradas por mujeres: contra la violencia de género; en favor de los derechos reproductivos; en defensa de los servicios de reproducción social; y en defensa de la salud de la clase trabajadora frente a la amenaza del COVID-19.

\section{“No estoy en el menú"}

Decenas de mujeres marchan tomadas del brazo y sus bocas están tapadas por una cinta que dice \#MeToo. No son actrices multimillonarias ni celebrities. Son trabajadoras de McDonalds, el martes 18 de septiembre de 2018 en la primera huelga contra el abuso sexual que logró coordinar acciones simultáneas en 10 ciudades norteamericanas: San Francisco, Chicago, Kansas City, Los Angeles, New Orleans, 
Missouri, Milwauke, St. Louis, Miami, Durham. Los principales reclamos de la protesta fueron: a) que la empresa creara comités anti acoso sexual (compuestos por mujeres trabajadoras, ejecutivos de la empresa, representantes de las franquicias y líderes de las organizaciones de mujeres); b) que se materialice la supuesta política de "tolerancia cero" que la empresa sostiene públicamente; c) que se lleven adelante en forma efectiva trainings para gerentes y empleados; $y d$ ) que se cree un sistema àgil para recibir y tramitar denuncias de situaciones de acoso sexual, que garantice que las denunciantes no serán castigadas 5 .

"Las trabajadoras de bajos ingresos no suelen tener acceso a los medios ni a los abogados que tienen las celebrities", dijo Eve Cervantez ${ }^{6}$, “Entiendo que es difícil para las celebrities hacer las denuncias [por acoso sexual], pero es importante saber que es mucho más difícil para las trabajadoras de bajos ingresos porque no cuentan con una red de contención para hacerlo"? Con esta sencilla frase, Cervantez iluminaba un hecho crucial: la profunda relación entre explotación y acoso sexual, o, dicho de otro modo, el acoso sexual como mecanismo de disciplinamiento de una fuerza de trabajo fuertemente precarizada, racializada, generizada y de carácter migrante ${ }^{8}$. Las trabajadoras de McDonalds describían el abuso sexual, la coerción y el acoso como hechos cotidianos en el lugar de trabajo $^{9}$, y denunciaban, además, las represalias sufridas por aquellas trabajadoras que se animaban a denunciar, bajo la forma de recorte de horas de trabajo (y de pago), cambios intencionales e inconvenientes en los horarios de trabajo y diversas formas de maltrato verbal.

La misma descripción podría haber sido hecha por una de Las $K$ ellys ${ }^{10}$ del otro lado del Atlántico en 2019. "En pelotas, me dijo que si me quedaba a pasar la noche me pagaba bien"11, denunció una trabajadora de limpieza de hotel organizada en Las Kellys en la campaña contra el abuso sexual que este colectivo de mujeres realizó en diversas ciudades del Estado Español. Como si fuera calcado del párrafo anterior, las trabajadoras de limpieza (camareras de piso) 
denuncian que sufren algún tipo de acoso sexual con frecuencia y exigen botón de pánico, limpiar las habitaciones de a dos (no solas) y no estar obligadas a realizar la limpieza cuando hay clientes adentro. Esto tiene que ver con que, a diferencia de lo que sucede en la cadena de comidas rápidas, lo que aquí se instala como parte de lo que sucede en el lugar de trabajo es el acoso por parte del "cliente" que "exige" favores sexuales como si fuera un ítem de los servicios prestados por el hotel. La campaña de 2019 logró tal fuerza, particularmente en las cadenas hoteleras 5 estrellas de las ciudades turísticas de la costa española, que obligó a la central sindical Comisiones Obreras (CCOO) de Canarias a tomar la demanda del abuso sexual bajo el lema "el cliente no siempre tiene la razón".

Muchos puntos en común tiene la huelga en McDonalds de 2018 y las acciones de Las Kellys en 2019: la sistematicidad del acoso sexual en los lugares de trabajo; el carácter precarizado de las condiciones de contratación y de trabajo; y el carácter inmigrante de muchas de las trabajadoras directamente relacionado con la precarización. Pero hay un cuarto componente que resulta importante: el tipo de organización que promueve la lucha a ambos lados del Atlántico. La jornada de huelga de McDonalds (que coordinó acciones en 10 ciudades) fue votada y dirigida por el Comité de Mujeres constituido por trabajadoras de distintos locales de comida rápida de distintas ciudades ${ }^{12}$. En el caso de Las Kellys también nos encontramos con una red creada por las mujeres trabajadoras de distintos hoteles de España, cuyo objetivo es luchar por las demandas de este sector precarizado de la fuerza de trabajo. Es decir, son organizaciones de mujeres trabajadoras que se construyen por fuera de los sindicatos pero con base en los lugares de trabajo, discuten sus necesidades como trabajadoras-mujeres y en esa discusión articulan lo que las organizaciones sindicales suelen disociar: las demandas laborales y las demandas de género, las cuales adquieren la forma de "demandas de clase". 


\section{"Aprendí sobre nuestros derechos como mujeres en la fábrica"}

En el pasillo central de la fábrica Madygraf se ve un cartel que dice "Domingo 22 de Julio, Madygraf se viste de verde: las mujeres al frente por el aborto legal y contra el ajuste". El verde es el color de la lucha por la legalización del aborto en Argentina y el cartel convoca a una asamblea de mujeres en el predio de la fábrica para preparar la participación en la jornada de lucha del 8 de agosto de 2018, día en que el Senado de la Nación tratará la Ley de Interrupción Voluntaria del Embarazo (IVE) ${ }^{13}$. Mady $\mathrm{Craf}^{14}$ es una empresa gestionada por sus trabajadores en la Zona Norte de Buenos Aires. El cartel fue discutido, votado y colgado por las mujeres trabajadoras que conforman La Comisión de Mujeres de la fábrica. Esta Comisión fue formada en 2011 (cuando la imprenta estaba aún bajo gestión de RR Donnelley) por las esposas, novias e hijas de los obreros, como organismo de apoyo a las luchas que llevaban adelante los trabajadores contra los recortes de la empresa, los intentos de despidos y los ataques a la Comisión Interna fabril. En ese entonces, la imprenta estaba compuesta solo por obreros varones. Con el paso del tiempo, la Comisión de Mujeres se transformó en uno de los pilares de la lucha de los trabajadores que desembocó en la toma y puesta en producción, y en uno de los pilares de la empresa bajo gestión obrera. Además de incorporar a las mujeres a las tareas de la imprenta, esta Comisión de Mujeres militante es el organismo que se ha ocupado de vincular con la lucha fabril como la lucha feminista que se despliega en Argentina más allá de las puertas de Madygraf. La asamblea del 22 de Julio convocó a más de 700 trabajadoras de diferentes establecimientos laborales de la zona y a militantes de la organización de mujeres Pan y Rosas ${ }^{15}$. Alli se votó: la participación activa en las manifestaciones planificadas para el día de la votación de la IVE en el Senado (8 de agosto), realizar una campaña para que en todos los lugares de trabajo se realicen asambleas para discutir el modo de apoyar la Ley de Legalización del Aborto, y exigir a las direcciones sindicales que declaren paro o asueto el 8 de agosto para que todas las mujeres puedan mo- 
vilizarse hacia las inmediaciones del Congreso de la Nación sin sufrir represalias por parte de las patronales.

Como es bien conocido, el debate por la legalización del aborto en Argentina conmovió al país y fue un punto de inflexión del masivo movimiento de mujeres desarrollado al calor de la consigna de \#NiUnaMenos en $2015^{16}$. Sin embargo, uno de los papeles más controvertidos fue el de la cúpula de la Confederación General del Trabajo (CCT $)^{17}$, la central obrera que concentra la mayoría de los trabajadores pertenecientes a ramas del sector privado. Unos días antes de que el proyecto de Ley fuera tratado en la Cámara de Diputados, algunos de los máximos dirigentes de la CGT firmaron una solicitada cuyo título era "Peronistas por la Vida", allí decían: “El aborto es un elemento ajeno a la cosmovisión justicialista, inescindible de la cultura del descarte". Un mes antes de que el proyecto de Ley fuera tratado en la Cámara de Senadores, la CCT sacó un comunicado institucional en el que afirmaba que no se posicionaría a respecto del aborto pero que alertaba al gobierno nacional sobre los problemas económicos que la legalización traería en el servicio de salud que administran los sindicatos debido a que aumentaría los costos de dicho servicio. El comunicado fue repudiado por dirigentes sindicales mujeres y por diversos sectores del movimiento feminista.

Esta política de la CGT hizo que se reforzara la frontera entre "cuestiones de mujeres" y "cuestiones de la clase trabajadora", abonando la concepción de que el aborto es un problema de decisión personal dirimible en el terreno de la moral individual (pro-choice vs. pro-vida), y no una cuestión que, siendo decisión de las mujeres y los cuerpos gestantes, afecta en forma directa las condiciones de reproducción del conjunto de la clase trabajadora. Esta ubicación de la cúpula de la CCT, hizo que la lucha por la legalización del aborto fuera (particularmente en el sector privado) un debate externo a la organización sindical en los lugares de trabajo. En aquellos sectores, como Madygraf, en los que la "ola verde" invadió los lugares de 
trabajo y sus organismos obreros, fue por iniciativa de la "minoría militante": comisiones de mujeres, militantes feministas, militantes políticas que comprenden el lugar de trabajo como parte de los territorios de lucha por los derechos de las mujeres.

\section{"Salvemos nuestras escuelas"}

Una multitud se manifiesta en el parquet que está en frente del capitolio del estado de West Virginia, en la ciudad de Charleston. Entre los muchos carteles que están en manos de los manifestantes, puede verse el que sostiene una niña: "Rosa Parks no estaba equivocada, tampoco lo están mis maestras". Estamos en una de las tantas movilizaciones que tuvieron lugar durante la huelga salvaje de nueve días ${ }^{18}$ llevada a adelante por las docentes de West Virginia, en lo que se conoció como el inicio la Primavera Docente (Teacher's Spring), ola de huelgas que se esparció por los estados de North Carolina, Kentucky, Arizona, Oklahoma y Colorado (EE. UU.) durante 2018.

La relación entre la icónica imagen de Rosa Parks y la huelga de West Virginia puede parecer exagerada, pero como describe Tithi Bhattacharya en sus reportes desde el lugar de los hechos ${ }^{19}$, lo que se respira entre los huelguistas es la sensación de estar dando el puntapié inicial para cambiar una situación de años y años de injusticia. Injusticia que no sólo los afecta como asalariados sino que afecta a todos aquellos para quienes la escuela pública es una institución irremplazable para la propia reproducción social. La huelga fue llevada a cabo por miles de docentes y personal de servicio de las escuelas públicas (choferes de colectivos escolares, cocineras, celadores, secretarias, etc.), pero involucró a otros miles de miembros de la comunidad que la apoyaron en forma activa. Por qué? Hay una serie de razones que pueden encontrarse en el libro de Eric Blanc Red State Revolt (2019), pero quiero detenerme en tres: a) La propia política de las organizadoras (mayoritariamente mujeres) de incorporar demandas que afectan al conjunto de los trabajadores como aumento de 
presupuesto para la PEIA (Public Employee Insurance Agency). Uno de cada siete habitantes de West Virginia depende de la PEIA para acceder a un sistema de salud. En este sentido, aunque la huelga de West Virginia puso en el centro el sistema educativo, en realidad fue una lucha en defensa de las dos grandes instituciones de reproducción social de la economía formal: la educación y la salud. De este modo, superó el corset sectorial y logró que su demanda interpelara al conjunto de la comunidad; b) El lugar de trabajo como espacio de toma de decisiones. Esto permitió, no sólo que las definiciones de los pasos a seguir se tomaran de forma democrática con votaciones escuela por escuela, sino también la posibilidad de que la auto organización de las docentes garantizara, por ejemplo, la provisión de comida para los estudiantes que dependen de la comida gratis en la escuela para su alimentación. Las docentes en huelga organizaron, junto con redes de madres, referentes religiosos y de organizaciones sociales (como comunidades de inmigrantes) un sistema de provisión de comidas de modo que la huelga no afectara la alimentación de los niños; c) El doble carácter de la escuela, como lugar de trabajo asalariado y como espacio fundamental de la reproducción social. En ese espacio dual, las demandas "clásicas" de una huelga laboral (como salario) se combinan con demandas profundamente obreras pero no laborales como la calidad de la educación y también del alimento que reciben las nuevas generaciones de trabajadores. Esa doble naturaleza de la escuela, como lugar de trabajo y lugar de "cuidado", agregado al carácter profundamente generizado que tiene la actividad docente (mayoritariamente llevada a cabo por mujeres), fueron elementos centrales para involucrar a la comunidad en la huelga. La huelga docente devino en una lucha por los medios y la calidad de la reproducción de la clase trabajadora y mostró el potencial de las trabajadoras de la reproducción social (mayoritariamente mujeres) como puentes entre los lugares de trabajo y los lugares de vida de la clase obrera, entre el ámbito de la producción y el de la reproducción. 


\section{"Nuestros cuerpos están en el frente de batalla"}

La foto recorrió las redes sociales ${ }^{20}$. Tres enfermeras paradas en la puerta del Hospital Mount Sinaí de Nueva York sostienen tres carteles. Uno dice "Servicio de salud de calidad para todos los neoyorquinos", otro dice "Cuántos de nosotros debemos morir? \#PPE [equipos de protección sanitaria para los trabajadores] por sobre las ganancias \#Protejan la primera línea". Y el tercero, que es el más grande, dice "Capitalismo: No Resucites. Trabajadores de la Salud por el Socialismo". La mujer que sostiene este cartel se llama Tre Kwon y es una enfermera de Nueva York, delegada de base de la Asociación de Enfermeras del Estado de Nueva York (NYSNA, por sus iniciales en inglés), y miembro del comité editorial del diario electrónico Left Voice ${ }^{21}$. La protesta fue el 3 de abril de 2020 y formó parte de una serie de manifestaciones protagonizadas por enfermeras y otros trabajadores de la salud en distintos hospitales de Nueva York, que en esos meses era el epicentro de la pandemia no sólo al interior de Estados Unidos sino a nivel mundial. Entre los hospitales en los que hubo manifestaciones en Nueva York se encuentra el Jacobi Medical Center y el Montefiori en el Bronx. Además, hubo protestas en hospitales de California, Florida, Kansas, Missouri, Nevada y Texas. En esas semanas, también salieron a luchar sectores de trabajadores de los centros logísticos y de almacenamiento: el 30 de marzo hubo manifestaciones en uno de los principales almacenes de Amazon, el JFK8, tras conocerse un caso de coronavirus entre los trabajadores; a inicios de abril, los trabajadores de Instacart llamaron a una huelga nacional exigiendo medidas de seguridad y pago adicional por pedido.

"Conformamos este equipo de tareas especial [Frontline Workers Task Force en el hospital Mount Sinai] porque vemos que todo ese discurso que sostiene Cuomo, Trump y otros politicos, pero también los CEOs, de que estamos todos juntos en esta guerra contra el COVID-19' es totalmente falaz. Somos nosotros los que ponemos nuestros cuerpos en la línea de fuego. Somos nosotros los que ponemos a 
nuestras familias en riesgo y nos ponemos en riesgo a nosotros mismos en nuestros trabajos. Por eso, estamos llamando la atención ahora"22. El mensaje de Kwon concentraba dos sentimientos que afloraron en distintos sectores de trabajadores con el correr de la pandemia. Por un lado, que mientras el discurso dominante hablaba de un virus que afectaba a todos, eran los trabajadores quienes estaban poniendo los cuerpos en el "campo de batalla”. Por otro, que más allá del demagógico reconocimiento a "los trabajadores esenciales" que circulaba en los grandes medios y en los discursos oficiales, la realidad del día a día en los lugares de trabajo era la de empresas que priorizaban la generación de ganancias por sobre la vida de las y los trabajadores a través de escatimar recursos para equipos de protección sanitaria para los trabajadores (PPE) y test de COVID-19, la extensión de la jornada de trabajo y el aumento del número de pacientes que cada enfermera debe atender, la no garantía de licencias pagas en caso de contagio, etc.

La excepcionalidad de la pandemia colocó sobre la mesa de debate tres elementos que suelen ser parte de "la morada oculta del Capital": a) la contradicción entre generación de ganancias y el cuidado de la vida de la clase trabajaora; b) la persistencia del lugar de trabajo como espacio en el que esa contradicción asume características extremas que afectan en forma directa los cuerpos de los trabajadores; c) el carácter "esencial" del trabajo de reproducción social asalariado y el protagonismo de las mujeres trabajadoras en él.

\section{Los lugares de trabajo como catalizador, las mujeres trabajadoras como puente}

Las luchas reseñadas más arriba tienen una serie de elementos en común. El primero y más evidente, es que son lideradas por mujeres. El segundo, es que las demandas exceden lo que clásicamente se entiende como lucha laboral, incorporando reclamos que aparecen como "extra laborales" como el fin de la violencia de género, la legalización del aborto, la defensa de la educación y la salud, y la prio- 
rización de la vida por sobre las ganancias empresarias. Es por eso que, como señalamos al inicio del artículo, caracterizamos estos casos como luchas de reproducción social en la medida en que están relacionadas con aspectos cruciales de las condiciones en las que la clase trabajadora lleva adelante su propia reproducción social. En tercer lugar, y muy importante, son luchas en el lugar de trabajo. Esto desafía, en cierto modo, uno de los sentidos comunes más asentados en los estudios del trabajo pero también en buena parte de la dirigencia sindical: la idea de que las luchas en el lugar de trabajo deben reducirse a los problemas relacionados con el consumo productivo de la fuerza de trabajo y con la retribución salarial de ese sector de trabajadores en ese lugar de trabajo. Esta concepción implica una doble reducción: una mirada sesgada sobre el lugar de trabajo y sus mecanismos de disciplinamiento (como hemos visto, el acoso sexual es parte de los mecanismos de disciplinamiento de una fuerza de trabajo generizada), pero supone también una incomprensión de la relación entre lo que pasa dentro del lugar de trabajo y lo que sucede fuera de él. En síntesis, construye una barrera infranqueable entre las esferas de la producción y la reproducción como si éstos fueran territorios dicotomizados para el capital. Las luchas lideradas por mujeres que hemos listado más arriba, desafían esta visión dicotomizada de los territorios de la lucha de clases: el lugar de trabajo no está tomado como un espacio sectorial sino como una posición de poder (otorgada por la capacidad para frenar la producción de ganancias a través de la acción coordinada de los trabajadores) que puede operar como un catalizador y un fortalecedor de demandas de la clase trabajadora como un todo.

Las contrarreformas neoliberales muestran que los ataques al conjunto de los trabajadores han sido tanto en el ámbito de la producción como en el de reproducción. La forma en que se ha dado la feminización de la fuerza de trabajo en las últimas décadas es un gran ejemplo de esto. Como señala Kim Moody en su análisis del modo en que el capital ha reconfigurado el "terreno de la guerra de clases" en los 
últimos 40 años (MOODY, 2017), la pauperización de los trabajadores (producto de la caída del salario real y relativo, y su consecuente empobrecimiento de las condiciones de vida) ha empujado a las mujeres al ingreso en el mercado de trabajo como nunca antes. Pero lo ha hecho generando nichos del mercado de trabajo en los que dicha ferminización se consolida: aquellos trabajos de menor carga horaria (y menores salarios) que permiten a las mujeres seguir llevando adelante el trabajo de reproducción no pago que realizan en el ámbito doméstico (especialmente las mujeres con hijos). Uno de esos nichos es lo que la OIT denomina "trabajos de cuidado" y nosotros denominamos "trabajos de reproducción social" en la economía formal (escuelas, hospitales, etc.), es decir, justamente esos sectores que han sido blanco de ataques de las políticas de ajuste y privatización neoliberales. Esto vuelve a las mujeres trabajadoras en "triples víctimas" del capitalismo neoliberal: como parte del conjunto de la clase trabajadora, porque han visto caer sus condiciones de vida y de trabajo sostenidamente en los últimos 40 años; como quienes Ilevan adelante (mayoritariamente) los trabajos asalariados de reproducción social, porque están en el centro de los ataques a los servicios de educación y salud públicos (y de las políticas para volverlos nichos de rentabilidad empresaria); como quienes llevan adelante (mayoritariamente) el trabajo de reproducción social no pago en el hogar porque han visto aumentar esta carga de trabajo debido al hecho de que cada escuela, jardín maternal, geriátrico, sala de salud, hospital que cierra o es privatizado, significa más trabajo para las mujeres de la clase trabajadora que no pueden pagar esos servicios en el mercado. En este sentido, las mujeres trabajadoras tienen una experiencia vívida de la profunda relación entre el ámbito de la producción y el de la reproducción, como los dos terrenos de ataque del capital. Esta posición específica de las mujeres al interior de la clase trabajadora se expresa de distintos modos en la Nueva Ola Feminista y permite pensar a las mujeres trabajadoras como puentes entre producción y reproducción. Pero también permite 
abrir el interrogante sobre los lugares de trabajo como catalizadores de luchas que no se reduzcan a objetivos parciales o sectoriales, sino que levanten demandas que interpelan al conjunto de la clase obrera en la medida en que refieren a necesidades centrales para su reproducción social, demandas no sólo relativas al género, sino también a la raza, la migración, la sexualidad.

\section{Bibliografía}

ARRUZZA, Cinzia. “From Women's Strikes to a New Class Movement: The Third Feminist Wave", Viewpoint, December 3, 2018. Disponible en https:// www.viewpointmag.com/2018/12/03/from-womens-strikes-to-a-newclass-movement-the-third-feminist-wave/. Acceso en: junio 2020

ARRUZZA, C. y BHATTACHARYA, T. "Teoría de la Reproducción Social. Elementos fundamentales para un feminismo marxista" en Revista Archivos de Historia del Movimiento Obrero y la Izquierda Na16, marzo, 2020. Disponible en https://www.archivosrevista.com.ar/numeros/index.php/archivos/article/view/251 Acceso en: marzo 2020.

ARRUZZA, C. y Varela, P. “Long Live the Women`s Committee”, Jacobin, 2018. Disponible en: https://www.jacobinmag.com/2019/04/argentina-worker-run-factory-feminism Acceso en: junio 2020.

BHATTACHARYA, Tithi (ed.) Social Reproduction Theory: Remapping Class, Re-Centering Oppression. Pluto Press: London, 2017.

BHATTACHARYA, Tithi “Bread and Roses in West Virginia”, Verso, 2018. Disponible en: https://www.versobooks.com/blogs/3669-bread-and-rosesin-west-virginia. Acceso en: junio 2020.

BLANC, Eric, Red State Revolt: The Teachers' Strike Wave and Working-Class Politics. Verso: London, 2019.

CAVALLERO, L.; GAGO, V.; VARELA, P.; VARON, C.; MITIDIERI, G. “Argentina's Anticapitalist Feminism", Jacobin, Septembre 2, 2018. Disponible en: https:// www.jacobinmag.com/2018/09/argentinas-anticapitalist-feminism. Acceso en: mayo 2020.

CISQUELLA, Georgina. Hotel Explotación: las Kellys, documentary, 2018. Disponible en: https://masdecultura.com/audiovisual/kellys/. Acceso en: mayo 2020. 
COLECTIVO NI UNA MENOS. Amistad política + inteligencia colectiva. Documentos y manifiesto 2015-2018, Buenos Aires, 2018.

FERGUSON, Susan "Las visiones del trabajo en la teoría feminista" en Revista Archivos de Historia del Movimiento Obrero y la Izquierda Na16, marzo, 2020. Disponible en: https://www.archivosrevista.com.ar/numeros/index. php/archivos/article/view/242. Acceso en: mayo 2020.

FERGUSON, Susan. Women and Work. Feminism, Labour and Social Reproduction. Pluto Press: London, 2020.

KWON, Tre "Only the Rank and File Can Save Us Now.0020Dispatches from the Frontlines of Care" Spectre Journal, April 15, 2020. Disponible en: https:// spectrejournal.com/only-the-rank-and-file-can-save-us-now/. Acceso en: abril 2020.

MARTÍNEZ, J. y BURGUEÑO, C. Patriarcado y Capitalismo: feminismo, clase y diversidad. Akal: Madrid, 2019.

MOODY, Kim. On New Terrain. How Capital is Reshaping the Class Battleground. Chicago: Haymarket, 2017.

ORLECK, Annalise. "MeToo and McDonalds"Jacobin, 2018. Disponible en: https://www.jacobinmag.com/2018/09/mcdonalds-strike-metoo-sexual-harassment-organizing. Acceso en: abril 2020.

RODRIGUEZ, Paula. Ni una menos. Editorial Planeta. Buenos Aires, 2016.

VARELA, Paula. "La reproducción social en disputa: un debate entre autonomistas y marxistas" en Revista Archivos de Historia del Movimiento Obrero y la Izquierda Na16, marzo, Buenos Aires, 2020. Disponible en: https://www. archivosrevista.com.ar/numeros/index.php/archivos/article/view/241. Acceso en: marzo 2020.

VARELA, Paula. "¿Existe un feminismo socialista en la actualidad? Apuntes sobre el movimiento de mujeres, la clase trabajadora y el marxismo hoy", en Revista Theomai Na39, primer semestre, Buenos Aires, 2019. Disponible en: http://revista-theomai.unq.edu.ar/NUMERO_39/1.\%20Varela.pdf. Acceso en: marzo 2020.

\section{Notas}

1 Doctora en Ciencias Sociales, Profesora de la Universidad de Buenos Aires e Investigadora del CEIL-CONICET, Argentina. https://orcid.org/0000-0002-16166633. paula.varela.ips@gmail.com 
2 Todas las citas textuales de artículos en inglés son traducciones de la autora.

3 Para profundizar en esta comprensión de la reproducción social véase el Dossier "El trabajo de las mujeres: feminismos, marxismos y reproducción social" publicado en la revista Archivos de Historia del Movimiento Obrero y la Izquierda $\mathrm{N}^{\mathrm{a} 16}$, marzo de 2020, con textos de Cinzia Arruzza y Tithi Bhattacharya (Arruzza y Bhattacharya, 2020) y Susan Ferguson (2020). Para un contrapunto entre esta visión de la reproducción y una visión autonomista, véase Varela (2020).

4 La feminización de la fuerza de trabajo incrementada en los últimos 40 años, muestra que uno de los principales nichos del mercado de trabajo en el que se han insertado las mujeres ha sido el trabajo de reproducción social asalarizado en los servicios públicos y en los privados.

5 Véase, Orleck, Annalise (2018)

6 Abodaga que representó a las 10 mujeres trabajadoras que realizaron la denuncia ante la Equal Employment Opportunity Commission en Mayo de 2018.

7 “McDonald's put under \#MeToo glare” https://enewspaper.latimes.com/infinity/ article_share.aspx?guid=14f39827-5f89-4b27-88bO-e08519fbdOdO

8 Las mujeres latinas y negras jugaron un rol protagónico en las protestas, en algunas ciudades los carteles eran bilingües: "McDonalds: Enought, no more sexual harassment. Basta, no más acoso sexual”.

9 Una encuesta realizada por Hart Research Associates (2016) dio como resultado que alrededor del $40 \%$ de las mujeres en esta rama de la industria alimenticia habían sufrido acoso sexual y se habían visto obligadas a aceptarlo porque no estaban en condiciones de perder sus trabajos. Además, 1 de cada 5 mujeres que denunció una situación de acoso, sufrió represalias por haberlo hecho: en algunos casos les bajaron la carga horaria o sus horarios de trabajo fueron modificados compulsivamente. https://hartresearch.com/wp-content/uploads/2016/10/ Fast-Food-Worker-Survey-Memo-10-5-16.pdf

10 Las Kellys es un acronismo de "las que limpian", https://laskellys.wordpress. com/2017/02/25/equipo-de-investigacion/. Distintas luchas de Las Kellys han sido recuperadas en el libro de Martínez y Burgueño (2019), y en el documental Georgina Cisquella (2018).

11 Véase “'Las Kellys' piden medidas de seguridad contra el acoso sexual”, La Sexta Journal, 28/10/2019.

12 Dicho comité se conformó en 2017, luego de la reunion anual que realiza la empresa todos los años. Según el caso, las protestas fueron apoyadas por distintos 
activistas de organizaciones como Fight for 15, MeToo, Tenant`s Unions, Time`s Up Legal Defense Fund, y miembros de organizaciones sindicales.

13 El Senado votó contra la IVE mientras más de un millón de mujeres se manifestaban en las calles reclamando la legalización.

14 La fábrica era propiedad de RR Donnelley, de origen norteamericano y con sede en Chicago. En 2014 lleva a cabo un lock out patronal, cerrando las puertas de la empresa y dejando al total de trabajadores desempleados. Los trabajadores y sus familias, ocuparon la fábrica y la pusieron a producer ese mismo año, conformando la cooperative de trabajo Madygraf. El nombre es en homenaje a Mady, la hija de uno de los principales dirigentes de la planta, Eduardo Ayala. Desde 2014 hasta la actualidad, la imprenta sigue funcionando bajo gestión obrera. https:// madygraf.com/. Véase Arruzza y Varela, 2019.

15 Véase http://www.laizquierdadiario.com/Pan-y-Rosas.

16 Veáse https://niunamenos.org.ar/. Colectivo Ni Una Menos (2018); Rodriguez, Paula (2016).

17 A diferencia de la CGT, los líderes de la Central de Trabajadores Argentinos (CTA), central obrera que concentra a los trabajadores estatales (administración pública nacional y provinciales, docents, salud, universidades, etc.) se posicionaron expresamente a favor de la legalización del aborto. Algunos de los sindicatos enrolados en la CTA tuvieron políticas activas en el lugar de trabajo, como es el caso de la AGTSYP (Asociación Gremial de Trabajadores del Subte y Premetro) que organizó lo que se conoció como la "Operación Araña", consistente en manifestaciones artísticas en las terminales del tendido de la red de subte de Buenos Aires.

18 La huelga comienza el 22 de Febrero y termina el 6 de marzo, lo que comprende 9 días hábiles.

19 Bhattacharya, Tithi (2018) “Bread and Roses in West Virginia”, https://www.versobooks.com/blogs/3669-bread-and-roses-in-west-virginia

20 Véase, "Only the Rank and File Can Save Us Now", Spectre Journal, April 15, 2020. https://spectrejournal.com/only-the-rank-and-file-can-save-us-now/. La foto fue tomada por Luigi W. Morris: https://www.facebook.com/luigisc.

21 https://www.leftvoice.org/

22 Véase, "Our Bodies Are On the Line", entrevista realizada por Amy Goodman para Democracy Now, April 3, 2020. https://www.democracynow.org/2020/4/3/ nyc_frontline_medical_workers_ppe_protest 


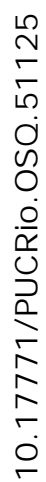

용 